\title{
SWIFT ULTRAVIOLET OBSERVATIONS OF SUPERNOVA 2014J IN M82: LARGE EXTINCTION FROM INTERSTELLAR DUST
}

\author{
Peter J. Brown ${ }^{1}$, Michael T. Smitka ${ }^{1}$, Lifan Wang ${ }^{1,2}$, Alice Breeveld ${ }^{3}$, Massimiliano de Pasquale ${ }^{4}$, \\ Dieter H. Hartmann ${ }^{5}$, Kevin Krisciunas ${ }^{1}$, N. Paul Kuin ${ }^{3}$, Peter A. Milne ${ }^{6}$, Mat Page ${ }^{3}$, and Michael Siegel ${ }^{7}$ \\ ${ }^{1}$ George P. and Cynthia Woods Mitchell Institute for Fundamental Physics \& Astronomy, Texas A. \& M. University, Department of Physics and Astronomy, \\ 4242 TAMU, College Station, TX 77843, USA \\ ${ }^{2}$ Purple Mountain Observatory, Chinese Academy of Sciences, Nanjing 210008, China \\ ${ }^{3}$ Mullard Space Science Laboratory, University College London, Holmbury St. Mary, Dorking Surrey, RH5 6NT, UK \\ ${ }^{4}$ Instituto di Astrofisica Spaziale e Fisica Cosmica di Palermo Via Ugo la Malfa 15390146 Palermo, Italy \\ ${ }^{5}$ Clemson University, Department of Physics \& Astronomy, Kinard Lab of Physics, Clemson, SC 29634-0978, USA \\ ${ }^{6}$ Steward Observatory, University of Arizona, Tucson, AZ 85719, USA \\ ${ }^{7}$ Department of Astronomy and Astrophysics, The Pennsylvania State University, 525 Davey Laboratory, University Park, PA 16802, USA \\ Received 2014 August 7; accepted 2015 March 16; published 2015 May 21
}

\begin{abstract}
We present optical and ultraviolet (UV) photometry and spectra of the very nearby and highly reddened supernova (SN) 2014J in M82 obtained with the Swift Ultra-Violet/Optical Telescope (UVOT). Comparison of the UVOT grism spectra of SN 2014J with Hubble Space Telescope observations of SN2011fe or UVOT grism spectra of SN 2012fr are consistent with an extinction law with a low value of $R_{V} \sim 1.4$. The high reddening causes the detected photon distribution in the broadband UV filters to have a much longer effective wavelength than for an unreddened SN. The light curve evolution is consistent with this shift and does not show a flattening due to photons being scattered back into the line of sight (LOS). The light curve shapes and color evolution are inconsistent with a contribution scattered into the LOS by circumstellar dust. We conclude that most or all of the high reddening must come from interstellar dust. We show that even for a single dust composition, there is not a unique reddening law caused by circumstellar scattering. Rather, when considering scattering from a time-variable source, we confirm earlier studies that the reddening law is a function of the dust geometry, column density, and epoch. We also show how an assumed geometry of dust as a foreground sheet in mixed stellar/dust systems will lead to a higher inferred $R_{V}$. Rather than assuming the dust around $\mathrm{SNe}$ is peculiar, SNe may be useful probes of the interstellar reddening laws in other galaxies.
\end{abstract}

Key words: dust, extinction - supernovae: general - supernovae: individual (SN2014J, SN2011fe, SN2012fr)

Supporting material: machine-readable table

\section{INTRODUCTION}

Type Ia Supernovae (SNe Ia) are important cosmological tools because their optical/near-infrared (NIR) luminosities are intrinsically bright and predictable. This means that their intrinsic brightness is correlated with distance-independent colors and light curve shapes (Phillips 1993, Riess et al. 1996, Goldhaber et al. 2001) so that they can be used as "standardizable" candles. Equally important (though perhaps implicit in being standard candles in multiple filters) is the fact that their colors are also predictable.

Understanding the colors allows one to infer the amount of dust extinction affecting the brightness in each of the filters based on the amount it preferentially extinguishes emission at shorter wavelengths, i.e., the amount of reddening. The amount of extinction (in magnitudes) at a certain wavelength or filter is often expressed as $A_{\lambda}=R_{\lambda} \times E(B-V) . R_{\lambda}$ is the extinction coefficient for that wavelength or filter. $E(B-V)$ is the differential extinction between the $B$ and $V$ filters, also called the color excess: $E(B-V)=A_{B}-A_{V}=(B-V)_{\text {observed }}-$ $(B-V)_{\text {intrinsic }} E(B-V)$ is often used to parameterize the amount of dust, though the observed value also depends on both the source spectrum and the shape of the extinction law (McCall 2004). The equation $A_{V}=R_{V} \times E(B-V)$ shows the relationship between the $B-V$ reddening and the extinction in V. Reddening in the Milky Way (MW) has an average value of $R_{V} \sim 3.1$ (Weingartner \& Draine 2001). A different extinction law could have a higher $R_{V}$ value, resulting in more extinction for the same amount of $B-V$ reddening. By definition, $R_{B}=R_{V}+1$, so extinction laws with higher $R_{V}$ are sometimes called shallower because of the smaller relative difference between $A_{B}$ and $A_{V}$. Conversely, extinction laws with lower $R_{V}$ have a relatively steeper change in the extinction with wavelength.

Curiously, a low value value for $R_{V}$ has been found for highly reddened individual $\mathrm{SNe}$ Ia, including $\mathrm{SNe}$ 1999cl: 2.01 (Krisciunas et al. 2006), 2003cg: 1.80 (Elias-Rosa et al. 2006), 2006X: 1.48 (Wang et al. 2008), and large samples of SNe Ia, including Tripp (1998): 2, Reindl et al. (2005): 2.65, Wang et al. (2006): 2.3, Conley et al. (2007): 1, Kessler et al. (2009): 2.18, Hicken et al. (2009): 1.7. The origin and implications of this unusual extinction law are uncertain. It could be due to small dust grains; such low $R_{V}$ are rare but have been observed in the MW (Gordon et al. 2003). Cardelli et al. (1989) parameterized a family of extinction curves as a function of $R_{V}$, but the smallest value of $R_{V}$ used was 2.6. So the use of smaller values is an extrapolation of the relationship between the reddening law shape and the $R_{V}$. Wang et al. (2009) find that when separated based on their expansion velocities, the absolute magnitudes of highvelocity (HV) SNe Ia tend to follow the steeper extinction law, while the others are more consistent with MW (i.e., $R_{V}=3.1$ ) dust. Foley et al. (2011) argue that it is only the highly reddened SNe Ia which follow the steep dust law, while HV SNe Ia have different intrinsic colors, and the less reddened samples of both 
Table 1

UVOT Photometry

\begin{tabular}{lcccccrrr}
\hline \hline SN & Filter & MJD & Mag & M_Err & MagLimit & SatLimit & Rate & R_Err \\
\hline SN2014J & UVW2 & 56679.4377 & 17.050 & 0.088 & 20.095 & 11.091 & 1.355 \\
SN2014J & UVM2 & 56679.4496 & NULL & NULL & 20.113 & 10.557 & 0.025 \\
SN2014J & UVW1 & 56679.4617 & 15.771 & 0.068 & 19.554 & 11.164 & 0.015 \\
SN2014J & U & 56680.5633 & 13.814 & 0.039 & 17.873 & 11.061 & 6.650 & 0.292 \\
\hline
\end{tabular}

Note. The photometry will also be available from the Swift SN website http://swift.gsfc.nasa.gov/docs/swift/sne/swift_sn.html.

(This table is available in its entirety in machine-readable form.)

populations can be fit with an extinction law similar to that of the MW.

Wang (2005) and Goobar (2008) show that circumstellar scattering will modify the "intrinsic" extinction law, resulting in a lower value of $R_{V}$. The extinction laws shown in Goobar (2008) were derived for a constant luminosity source. The effective extinction law for a time-varying SN would be more complicated and depend on the dust geometry and density as well as the intrinsic emission from the SN (Wang 2005; Amanullah \& Goobar 2011). But the scattering of light could result in a smaller value of $R_{V}$.

Ultraviolet (UV) emission is very sensitive to the dust grain size and geometry. While the amount of extinction in the midUV is less dependent on the grain size when normalized by the color excess $E(B-V)$ (Cardelli et al. 1989), the relative extinction compared to the optical is very dependent on the grain size. For the circumstellar scattering case, the wavelength dependence of scattering and absorption steepens the effect of extinction in the UV (Wang 2005; Goobar 2008; Brown et al. 2010). This is because the albedo for MW/LMC type grains peaks in the optical, reducing the extinction in the optical while increasing $E(B-V)$ for the same amount of dust. Observations on either side of the albedo bump should reveal whether the extinction law shapes are due to dust variations or circumstellar scattering.

The high sensitivity of UV photons to extinction makes them a good probe of the extinction laws, but it also makes them very hard to detect. Once there is enough dust (of whatever form or geometry) to make a significant impact on the optical colors, the UV light is almost completely extinguished. It is a challenge to detect extinguished UV emission at the distances at which $\mathrm{SNe}$ are typically discovered. The discovery of the very nearby, and highly reddened, SN 2014J in M82 (Fossey et al. 2014) provides a unique opportunity to study the effect of heavy extinction on the UV light. However, we will show that measuring these effects is difficult in the UV, especially with broadband filters.

In Section 2 we present UV/optical photometry and spectroscopy of SN 2014J obtained with the Ultra-violet/ Optical Telescope (UVOT; Roming et al. 2005) on the Swift satellite (Gehrels et al. 2004). We compare different extinction laws to the observed spectra in Section 3. Section 4 describes our circumstellar model and Section 5 compares it to the observed photometry. In Section 6 we show how the assumption of dust extinction as a foreground screen increases the inferred $R_{V}$. We summarize these results in Section 7.

\section{SWIFT OBSERVATIONS OF SN 2014J IN M82}

The Swift satellite started observing SN 2014J (Cao et al. 2014; Fossey et al. 2014) in M82 at 2014 January 22.43 UT. Early Swift results were reported by Brown \& Evans (2014). Photometry observations were made on a roughly daily basis between January 22 and February 25 and then continuing with a lower cadence until April 10. Because the UVOT works as a photon detector, bright sources suffer from coincidence loss-multiple photons arriving during the same frame are undercounted (see Poole et al. 2008 for more details and the correction method). Because of the brightness of the $\mathrm{SN}$ in the optical, special "hardware window" modes were used to more frequently read out a smaller section of the detector to reduce the effect of coincidence loss due to the bright $\mathrm{SN}$ and the relatively bright underlying galaxy. Because of the faintness in the UV additional observations were made to increase the exposure time in the uvm2 filter. The filter characteristics are described in Poole et al. (2008) and Breeveld et al. (2011), but will be described in detail below. The host galaxy was imaged repeatedly by UVOT in the years prior to explosion (Hutton et al. 2014). These images were used to subtract the count rates of the underlying galaxy.

Observations with the UVOT's UV grism were made at seven epochs before and around maximum light (though each epoch is broken up into separate exposures due to the observability windows). The time ranges and total exposure times used here are listed in Table 3. We also use the Swift/ UVOT grism spectra of SN 2012fr for comparison purposes (PI: R. Foley). SN 2012fr was a very broad near-UV (NUV)$\mathrm{red} /$ irregular SN with high velocity features. We use a distance modulus of $31.18 \pm 0.05$ derived from Cepheid observations (Freedman et al. 2001). The observations used are also listed in Table 3.

\subsection{Photometric Data Reduction}

Swift UVOT data were analyzed using the reductions methods for the Swift Optical/UV Supernova Archive (Brown et al. 2014a) including the revised UV zeropoints and timedependent sensitivity from Breeveld et al. (2011). The photometry is given in Table 1 . The six filter light curves are displayed in Figure 1.

Because of the brightness of the $\mathrm{SN}$ and the underlying galaxy, we do not use the normal full-frame images in the optical but only those taken using a hardware window to increase the frame rate (by reading out a smaller section of the detector) and reduce coincidence losses. By comparison with well calibrated SN photometry from ground based observations with linear detectors, Brown et al. (2009) found the coincidence loss correction to yield accurate magnitudes in the full field mode when the underlying galaxy count rate is less than about 5 counts $\mathrm{s}^{-1}$ in a $5^{\prime \prime}$ aperture. The underlying galaxy count rates in $\mathrm{b}$ and $\mathrm{v}$ are more than five times the brightness limit for the full-frame images, so the factor of four gained by using the smaller readout windows is not enough in 


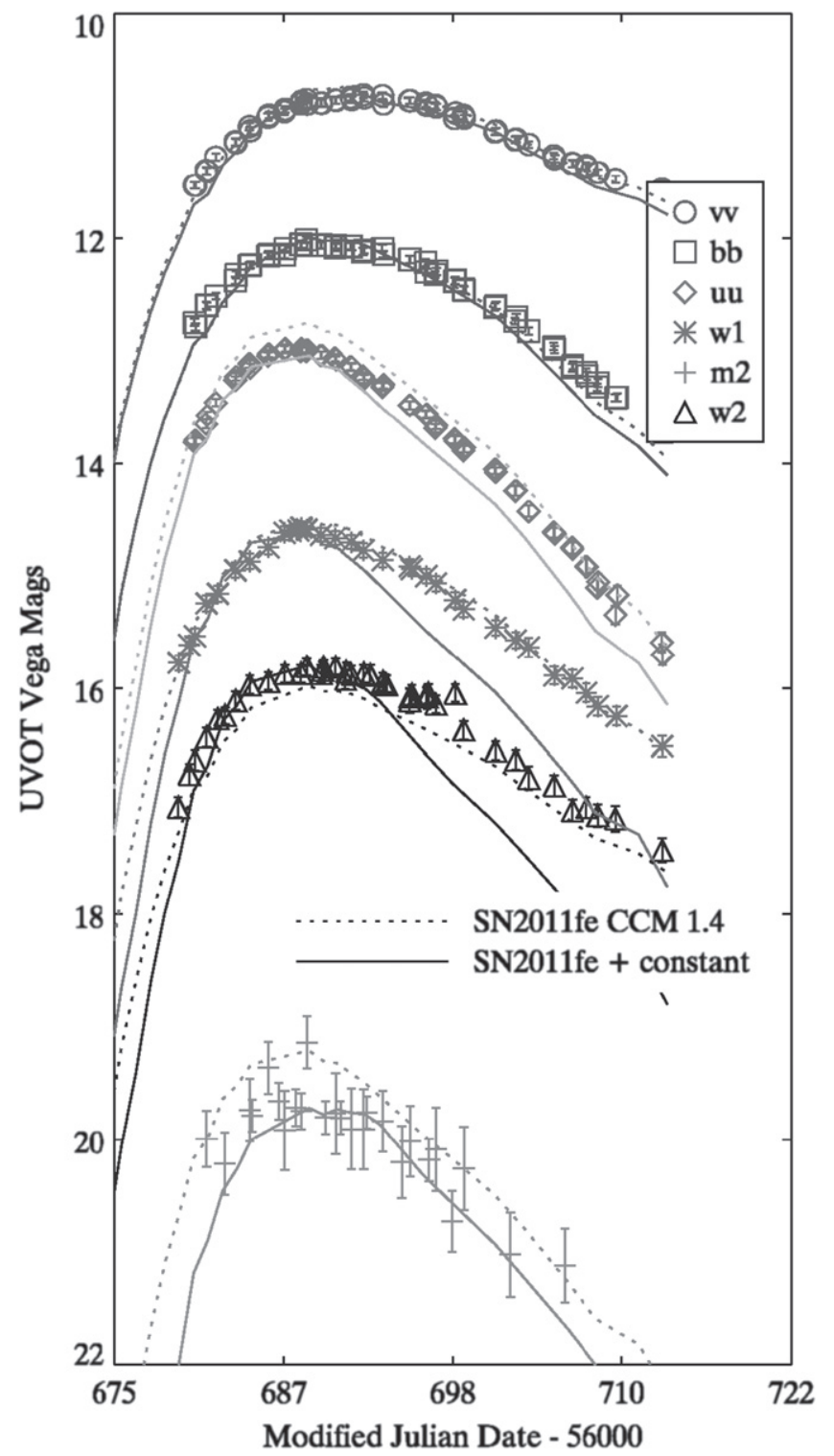

Figure 1. UVOT light curves of SN2014J given in observed Vega magnitudes versus time. The reddening naturally separates the curves with no arbitrary offsets needed. We overplot the spectrophotometric light curves for SN $2011 \mathrm{fe}$ (based on bolometric spectra from Pereira et al. 2013) without extinction (but offset to match the peak magnitudes). We also plot spectrophotometric light curves for SN 2011fe after reddening with a CCM extinction law with $E(B-V)=1.4$ and $R_{V}=1.4$ and correcting only for the difference in distance moduli.

this case. The optical bv data is found to be about $0.2 \mathrm{mag}$ fainter at peak than that found by Tsvetkov et al. (2014) and Marion et al. (2015). The count rates of the host galaxy in the other UVOT filters are much lower than the optical and thus the photometry should not be significantly affected. In the rest of the paper we use the $B$ and $V$ photometry from Marion et al. (2015) which is similar to the UVOT bv photometry of other $\mathrm{SNe}$ against which we have compared (Poole et al. 2008; Brown et al. 2009). In many instances we reference the time of observations with respect to the time at which the $\mathrm{SN}$ reached maximum light in the B band $\left(t_{\mathrm{Bmax}}\right)$. We use the SNooPy fit results from Marion et al. (2015) giving the time of maximum light as MJD 56689.74 (2014 February 1.74). For SN 2014J we use a distance modulus of $27.73 \pm 0.02$ derived from color-
Table 2

Light Curve Parameters

\begin{tabular}{lccc}
\hline \hline Filter & $\begin{array}{c}\text { Peak Magnitude } \\
(\mathrm{mag})\end{array}$ & $\begin{array}{c}\text { Peak Date } \\
(\mathrm{MJD})\end{array}$ & $\begin{array}{c}\text { Delta M15 } \\
(\mathrm{mag})\end{array}$ \\
\hline uvw2 & $15.800 \pm 0.048$ & $56688.9 \pm 1.1$ & $0.98 \pm 0.11$ \\
uvm2 & $19.54 \pm 0.18$ & $56687.9 \pm 0.5$ & $0.95 \pm 0.21$ \\
uvw1 & $14.594 \pm 0.023$ & $56688.0 \pm 0.4$ & $0.88 \pm 0.07$ \\
u & $12.983 \pm 0.014$ & $56687.4 \pm 0.2$ & $1.14 \pm 0.03$ \\
\hline
\end{tabular}

Note. $\mathrm{b}$ and $\mathrm{v}$ fit parameters excluded due to the data being corrupted by high galaxy count rates.

Table 3

Swift/UVOT Grism Exposures for SNe 2014J and 2012fr

\begin{tabular}{lccc}
\hline \hline $\begin{array}{l}\text { SN Phase } \\
\text { (Days) }\end{array}$ & Observation ID+extensions & $\begin{array}{c}\text { Date } \\
(\mathrm{UT})\end{array}$ & $\begin{array}{c}\text { Exposure } \\
(\mathrm{s})\end{array}$ \\
\hline SN2014J-1 & $33123025+2-6$ & $\begin{array}{c}\text { 2014 Jan 31- } \\
\text { 02/01 }\end{array}$ & 7518 \\
& & 2014 Feb 02-3 & 8030 \\
SN2014J 0 & $33123032+2-4$, & & \\
& $33123033+1-4$ & 2014 Feb 04 & 4330 \\
SN2014J+2 & $33123036+2,33123037+2$ & 2014 Feb 05 & 4730 \\
SN2014J+3 & $33123038+1-2$, & 2012 Nov 11 & 4545 \\
SN2012fr-1 & $33123040+1-2$ & 2012 Nov 13 & 16425 \\
SN2012fr+1 & $32614021+1-3$, & & \\
\hline
\end{tabular}

magnitude fitting of the tip of the red giant branch (Jacobs et al. 2009).

To measure the light curve parameters in the UV filters, the light curves have been fit by stretching appropriate templates to the observed data. The three UV filters are fit with the UVOT templates from SN 2011fe (Brown et al. 2012) and the u band is also fit using the uvw1 template. The resulting peak times, peak magnitudes, and the change of magnitude in the 15 days after maximum light are reported in Table 2 . The $\mathrm{b}$ and $\mathrm{v}$ are not reported due to the possible coincidence loss issues. Milne et al. (2010) studied the UV light curve shapes of a large number of Swift SNe Ia. The UV light curves of SN 2014J are broader than all those in Milne et al. (2010) with the u band comparable only to SN 2005cf. SN 2011aa is comparably broad (Brown et al. 2014b). As shown in Figure 1, this broadening is consistent with the extinction causing a photon distribution biased to the redder photons (which fade slower) within each band. This is discussed in more detail in the Appendix.

\subsection{Grism Spectral Reduction}

Grism observations of SN 2014J and SN 2012fr were extracted using the default parameters of the UVOTPY package (Kuin 2014; Kuin et al. 2015). ${ }^{8}$ The nominal wavelength accuracy is $20 \AA$ and the flux calibration is accurate to about $10 \%$. Second order contamination is small for unreddened, UV-faint SNe Ia and considered negligable in this case. The exposures from each day (corresponding to the ExposureID's in Table 3) were wavelength shifted to agree with a master frame using a chi square minimizing wavelength shift routine. Next, they were coadded using variance weighting to create a single spectrum for each obsid. The SN $2012 \mathrm{fr}$ spectra were then wavelength shifted to agree with a

\footnotetext{
www.mssl.ucl.ac.uk/www_astro/uvot
} 

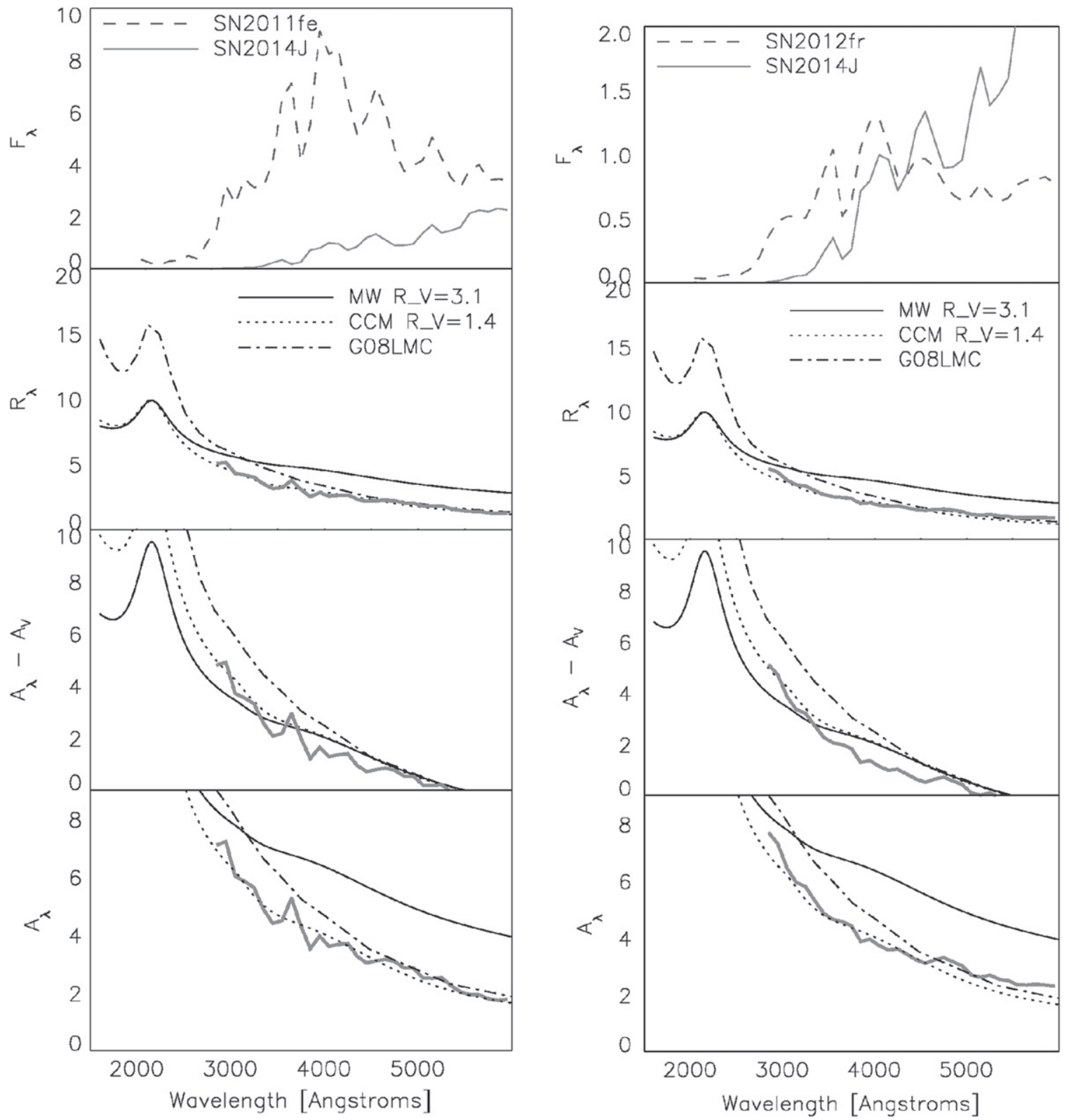

Figure 2. Top left panel: combined spectra of SN 2014J and 2011fe smoothed to $100 \AA$. The spectra have been corrected for the estimated extinction from the Milky Way. Lower left panels: the spectrum of SN 2014J divided by the spectrum of SN 2011fe and corrected for the relative distances to mimic $R_{\lambda}\left(\mathrm{i} . \mathrm{e}\right.$., $A_{\lambda} / E(B-V)$ ), $A_{\lambda}-A_{V}$, and $A_{\lambda}$. These are compared to the corresponding functions for various extinction laws. The best match is with the Milky Way law with $R_{V}=1.4$. Right panels: same as left but comparing to SN 2012fr.

maximum light spectrum of 2012fr from Childress et al. (2013) obtained from the WISEREP database (Yaron \& GalYam 2012) between wavelengths of 3500-5500 Å. For comparison of the spectra near maximum-light, SN 2014J spectra between February 1 and 5 were combined into a single spectrum as were both SN 2012fr spectra. The combined maximum light spectra are displayed in the top panel of Figure 2.

\section{COMPARING EXTINCTION LAWS TO THE MAXIMUM LIGHT SPECTRA}

The extinction law, by which we mean the wavelength dependence of extinction, has been measured to many lines of sight in the MW, the LMC, and the SMC. Cardelli et al. (1989; CCM) parameterized the different shapes of the extinction law by the corresponding $R_{V}$. In this work we will consider extinction laws with $R_{V}=3.1$ corresponding to the average value of the MW, as well as other values of $R_{V}$, in particular $R_{V}=1.4$, the best fit value from Amanullah et al. (2014). These will be called "MW 3.1" and "CCM 1.4" hereafter. They correspond to different slopes in the optical and NUV. Both feature the " $2175 \AA$ " bump, whose strength (as measured by $R_{2175 \AA}$ ) is largely independent of $R_{V}$ (Cardelli et al. 1989). We also compare with an LMC extinction law modified by the effects of circumstellar scattering (for a steady state source, dubbed G08LMC) from Goobar (2008). Circumstellar scattering will be discussed in more detail below.

To infer the wavelength dependent extinction, one needs a comparison object with similar intrinsic colors. To first order, $\mathrm{SNe}$ Ia appear to follow a single-parameter continuum 
determined by the ${ }^{56} \mathrm{Ni}$ yield. The observed parameter typically associated with this is the light curve width or the decay parameter $\Delta m_{15}(B)$, which is a measurement of the magnitudes the B-band light curve decays in the 15 days after maximum light. One would typically compare SNe Ia with similar values of $\Delta m_{15}(B)$. This is somewhat complicated by the extinction because the evolving spectral shape results in a different decay rate when extinguished. Phillips et al. (1999) give this correction as: $\Delta m_{15}(B)_{\text {true }}=\Delta m_{15}(B)_{\text {obs }}+0.1 \times E(B-V)$. Tsvetkov et al. (2014) report a $\Delta m_{15}(B)=1.01$ and $E(B-V)=1.3$ for $\mathrm{SN} 2014 \mathrm{~J}$, resulting in an estimated reddening corrected $\Delta m_{15}(B)=1.14$.

SN 2011fe is a SN with $\Delta m_{15}(B)=1.108$ (Munari et al. 2013) with excellent multi-wavelength observations, e.g., from Brown et al. (2012), Hsiao et al. (2013), Pereira et al. (2013), and Mazzali et al. (2014). Amanullah et al. (2014), Foley et al. (2014), and Goobar et al. (2014) found it to be a good comparison for SN 2014J. We use a distance modulus of $29.04 \pm 0.20$ from Shappee \& Stanek (2011). We also compare it to SN 2012fr, which is a broad SN Ia $\Delta$ $m_{15}(B)=0.8$ (Zhang et al. 2014) with HV spectral features (Childress et al. 2013).

To determine the observed reddening law we use a maximum light Hubble Space Telescope (HST) spectrum for SN 2011fe Mazzali et al. (2014), a combined maximum light UVOT spectrum for SN 2012fr, and the combined maximum light UVOT spectrum for SN 2014J. We smooth the spectra by rebinning them to $100 \AA$ resolution while conserving the flux. The smoothed spectra are shown in the top panels of Figure 2, with SN 2011fe on the left and SN 2012fr on the right. To determine the extinction difference as a function of wavelength we correct for distance and divide the flux of the reference spectrum by the flux of SN 2014J and convert it to an extinction in magnitudes. The wavelength dependence of extinction can be visualized in different ways, so in the lower panels we show $R_{\lambda}$ (i.e., normalizing by $E(B-V)$ to focus on the shape), $A_{\lambda}-A_{V}$ (to focus on the slope differences), and $A_{\lambda}$ (the total effect). In all panels we show the respective values for the various extinction laws using $E(B-V)=1.4$, the approximate color excess of SN 2014J from Amanullah et al. (2014). One could also correct to $A_{V}$ or any other parameter.

The observed extinction laws, as determined by both comparison spectra, are very similar. The one derived from SN 2011fe shows more structure due to the large velocities in SN 2014J. In all plots, but best seen in terms of $A_{\lambda}$ in the bottom panels, the CCM 1.4 law gives the best match. The G08LMC law is the next best fit, though with the measured extinction trending lower than it shortward of $4000 \AA$. Better fits might be obtainable with the CCM $R_{V}$ (Cardelli et al. 1989) and power-law (Goobar 2008) parameterizations, but here we were trying to test the fit of previously used extinction laws. Deviations could result from the difference between the real and model extinction curves or the true, unreddened SN and the SN model.

\section{CIRCUMSTELLAR SCATTERING MODEL}

Many lines of evidence show that the reddening law to SN 2014J has a low value of $R_{V}$. These include the photometric colors shown above from Swift/UVOT as well as those of HST (Amanullah et al. 2014). The NUV/optical spectroscopy in Section 3 and Foley et al. (2014) and optical spectroscopy from (Goobar et al. 2014) also show a low $R_{V}$. A low value of $R_{V}$ could be caused by smaller dust grains (Weingartner \& Draine 2001) or from certain geometries of circumstellar dust (Wang 2005; Goobar 2008). One key difference is in the temporal changes in the extinction magnitudes and colors. In the case of interstellar scattering from dust grains, the only temporal changes are due to the changing spectral shape. $R_{\lambda}$ will change modestly as the spectral shape changes (Phillips et al. 1999; Wang 2005). In the case of circumstellar scattering, however, photons are scattered back into the line of sight (LOS) with a delay time proportional to the distance to the scattering dust (Wang 2005; Amanullah \& Goobar 2011). As SN 2014J is shown to have a very low value of $R_{V}$ (Amanullah et al. 2014; Foley et al. 2014 and above), we now test the other predictions of scattering to see if they are also consistent.

For extinction from a foreground cloud or sheet of dust, scattering and absorption both remove photons from the LOS, and the flux is scaled by the factor $e^{-\tau}$, where tau is the optical depth due to scattering and absorption. For a cloud around the source, just as much light is scattered back into the LOS as is scattered out of it. The fraction of the light lost which is lost to scattering is given by the albedo $\omega$. In the single scattering approximation (each photon can be absorbed or scattered at most once) this scattered flux is added back in and only the absorbed photons are lost. The observed flux $\left(F_{\text {obs }}\right)$ is thus related to the intrinsic flux $\left(F_{\text {int }}\right)$ as follows:

$$
F_{\text {obs }}=F_{\text {int }} \times\left(e^{-\tau}+\omega \times\left(1-e^{-\tau}\right)\right) .
$$

In the analysis below we use the wavelength-dependent values ${ }^{9}$ of $\tau$ and $\omega$ consistent with average properties of LMC dust Weingartner \& Draine (2001). We will refer to this single scattering extinction law as SSLMC below. For low values of $\tau$ this is consistent with the analytic expression used by Wang (2005) and the Monte Carlo determined law from Goobar (2008) for the same LMC type dust. We point out that the power law parameterization of Goobar (2008) is not directly predicted from circumstellar scattering but is a good fit to the region longward of the $2175 \AA$ bump. Because the albedo peaks in the optical, the extinction is reduced much more in the optical than in the UV.

At higher values of optical depth the single-scattering approximation breaks down. To account for multiple scattering in an analytic way, we use Equation (9) of Mathis (1972) for the fractional flux emitted by the circumstellar shell around an embedded star. This is given as

$$
F_{S}=1.1\left[1-e^{(-\tau)}\right] \times \omega \times e^{\left[-\tau(1-\omega)^{0.5} \times(1-g)^{0.3}\right.} .
$$

Here $g$ is the scattering phase function, which determines the relative fractions of photons scattered into different angles. The total flux observed is then

$$
F_{\mathrm{obs}}=F_{\mathrm{int}} *\left[e^{-\tau}+F_{s}\right]
$$

where the first term is the extinguished light directly from the source and the second is the contribution of scattering from the nebula. This is labeled MSLMC below. For low values of optical depth this curve matches the shape of the W05 and G08 laws above $3000 \AA$. These modifications of the LMC law are also compared in the top panel of Figure 3. In our

\footnotetext{
https://www.astro.princeton.edu/ draine/dust/dustmix.html
} 
implementation, the column density of hydrogen (nh) is given as an input to calculate $\tau$ from the cross sections given by Weingartner \& Draine (2001).

Several groups have used the power-law parameterization of Goobar (2008) and found it consistent with the observed optical and NIR colors of highly-reddened SNe. In such instances the Goobar (2008) parameterization is used almost exclusively as a reddening law-namely a wavelength dependence of the extinction. However, those scattering laws shown in the top panel of Figure 3 are applicable for circumstellar scattering of a source with a constant luminosity/spectrum. As such they cannot be applied to a time-variable source and be considered as a scattering model. The delay time dependence of the scattering is a critical element of such a model with strong observational consequences (Wang 2005; Amanullah \& Goobar 2011).

To evaluate the temporal evolution of the scattering, we expand the model used in Wang (2005). We use the Pereira et al. (2013) UV/optical spectral series of SN $2011 \mathrm{fe}$ interpolated to a $10 \AA$ resolution so that that accurate spectrophotometry can be done on the reddened/scattered spectra taking the full filter bandpasses into account. Given a dust shell radius (in $\mathrm{cm}$ ) and optical depth (calculated from the number of hydrogen atoms along the path, $n_{h}$ ), the light travel time to the dust shell and back into the LOS is calculated in 500 radial directions between $0^{\circ}$ and $180^{\circ}$. For each time step in the spectral series, the spectrum at the appropriate time in the past is interpolated. The flux from the shell escaping into the LOS is calculated using Equation (9) of Mathis (1972), taking into account the scattering angle, optical depth, and albedo. For each direction bin the Henyey \& Greenstein (1941) function is used to determine the fraction of scattered photons which are redirected to the observer. These time-delayed contributions are integrated over a $360^{\circ}$ rotation to cover the whole sphere. While the largest time delay is twice the light travel time to the dust sphere (photons traveling directly away from the observer and being scattered back), the largest solid angle has half that time. Photons are not scattered isotropically, and the forwardscattering nature of the LMC dust decreases the median delay time. The contribution from scattered photons is added to the extinguished spectrum for that epoch. This creates a model spectral series representing the observable spectra from a SN like SN 2011fe and the input dust density and geometry.

By comparing the input and output spectral series, we can determine the effective reddening law at any epoch. The middle two panels of Figure 3 show the reddening laws at maximum light for the SN2011fe template for different radii and column densities. In Goobar (2008) the extinction law was considered insensitive to the size of the scattering cloud (with a change in density allowing for the same optical depth). For a timevariable source, however, the distance to the cloud directly affects the time delay in the scattered photons which will contribute to the observed light curve. Figure 4 of Wang (2005) shows the change in $R_{B}^{0}$ at maximum light as a function of the dust radius. The low values of $R_{V}$ attributed to circumstellar scattering only occur if the dust is quite close $\left(<10^{17} \mathrm{~cm}\right)$. The shape of our extinction curve also changes with column density, while Goobar (2008) showed $R_{V}$ to be constant with $E(B-V)$. We find the shape of the extinction curve to be similar in the optical but differ in the UV. The differing shape is due to different fractions of unabsorbed and scattered light. The bottom panels of Figure 3 shows the effect of this scattering on the effective extinction law at different epochs. Here the extinction curves are separately normalized by the color excess at each epoch or, in the bottom plot, at maximum light. $R_{V}^{0}$ is the notation used by Wang (2005) to designate $A_{V}$ divided by $E(B-V)_{\text {peak }}$ rather than the color excess at the same epoch. Clearly the reddening decreases with time as the scattered photons from the light curve peak add to the SN flux. While we consider here only a thin shell, the width of the shell will also have an effect (Amanullah \& Goobar 2011). The general trends of Wang (2005) and Goobar (2008) are reproduced, namely a smaller value of $R_{V}$ are seen in some of the models with a smaller radius where the scattering delay is neglible. However, it is clear that a single extinction law cannot be considered representative of circumstellar interaction as it varies with distance, density, and the intrinsic spectral evolution as well as phase. We have only considered a thin shell-other geometries give qualitatively similar results (Amanullah \& Goobar 2011) but which may need to be tested for best results.

\section{SN 2014J COMPARED TO CIRCUMSTELLAR SCATTERING MODELS}

\subsection{Comparison Between Expected and Observed Extinction Evolution}

One prediction of the circumstellar scattering model is a negative temporal change in $\mathrm{A}_{\lambda}$. This is due to the time delay of the scattering and the rising flux of the SN. Thus the early light curve will suffer from subtractive extinction but as time passes there should also be an additive component from the scattered light which grows with time until the light curve peak has been scattered back into the LOS. The SN light curve will be broader than it would be if observed in isolation. When compared to an unreddened $\mathrm{SN}$ this will appear as a reduction in the $\mathrm{A}_{\lambda}$ as the SN appears less extinguished with time (see Figure 2 in Wang 2005). We have already shown in Figure 1 that applying a uniform reddening law with low $R_{V}$ to the SN 2011fe template does a good job of matching the SN 2014J. This includes a stretching out of the light curves consistent with the changing effective wavelengths compared to the unreddened SN 2011fe light curves (as also shown by Amanullah et al. 2014).

The UV/optical spectral series of SN 2011fe allows us to directly compare the differences between objects to the magnitude differences caused by extinguishing the evolving spectrum. In Figure 4 we show with dotted lines the magnitude differences between the reddened and unreddened SN $2011 \mathrm{fe}$ spectrophotometry. The evolution is modest in the optical but is quite significant in the NUV. The plotted symbols show the difference between SN 2014J and the SN2011fe spectrophotometry interpolated to the same epochs. The difference shows a modestly greater slope than expected from pure spectral evolution. The difference peaks near maximum light and is smaller at early and late times. It does not exhibit the rapid fading predicted by the circumstellar scattering (Wang 2005) but is characteristic of SN 2014J having slightly broader light curves than SN $2011 \mathrm{fe}$ (corresponding to a relative stretch factor of 1.1).

To get the temporal decrease in $\boldsymbol{A}_{\lambda}$ expected from circumstellar scattering (Wang 2005), one must assume an intrinsically broader SN as the template. We simulate this in Figure 4 by stretching the SN2011fe template before the 


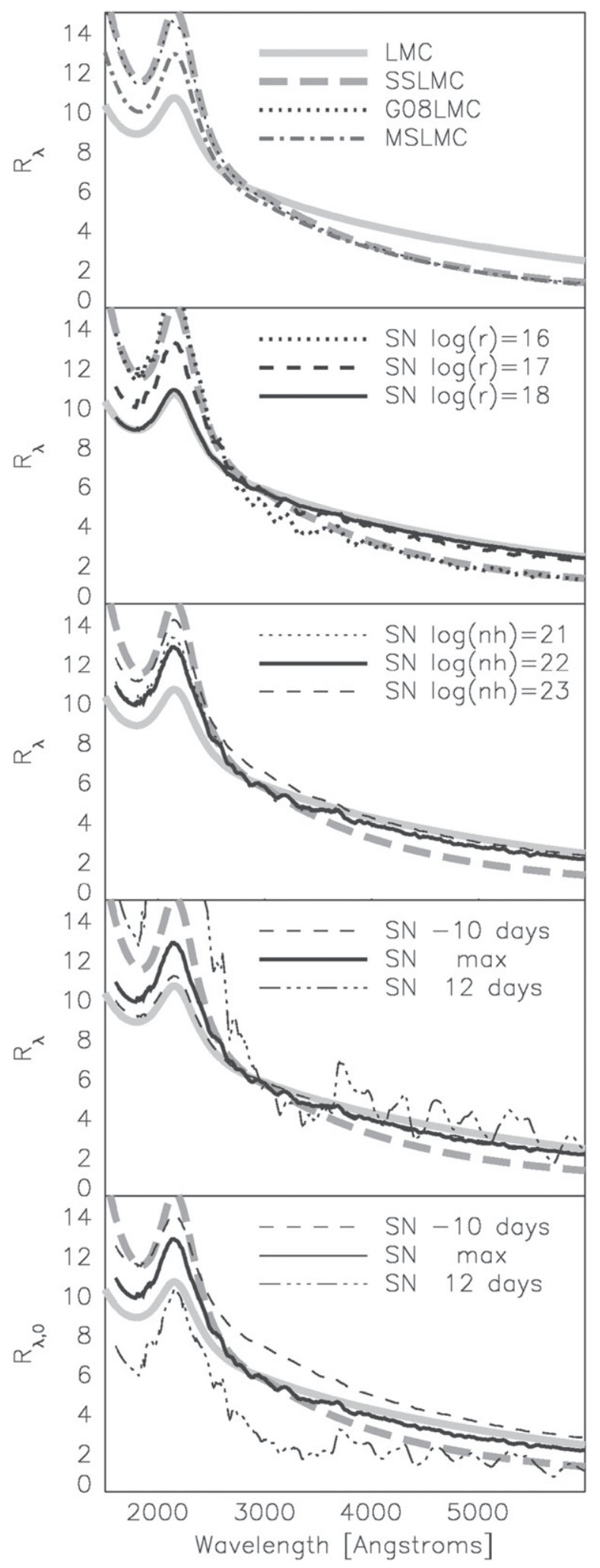

Figure 3. Top panel: extinction law for the LMC compared to a single scattering model (SSLMC), the G08 model, and a multiple scattering model (MSLMC). Lower panels: the effective extinction laws are shown for different radii (in $\log (\mathrm{cm})$ at maximum light with a constant optical depth), hydrogen column density (at maximum light with a constant radius of $10^{17} \mathrm{~cm}$ ), and epochs (in days from maximum light, with a constant radius and optical depth). The LMC and SSLMC models are shown in the lower panels as well for comparison.

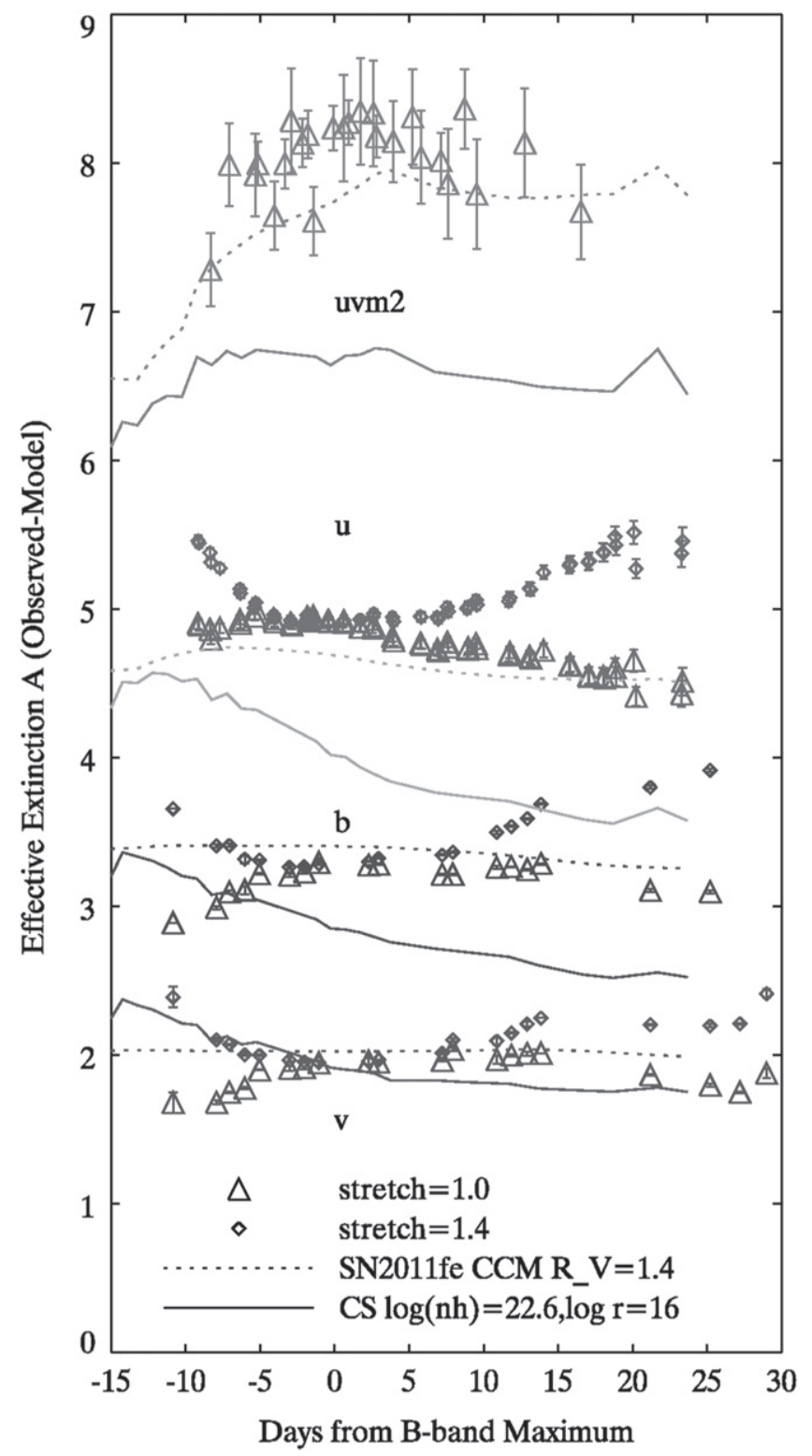

Figure 4. Distance-corrected magnitude differences between SNe 2014J and 2011fe (triangles) and between SN 2014J and a stretched SN 2011fe. We label the $y$-axis "Effective Extinction" because the differences may or may not be due wholly to the extinction. The dotted lines represent the magnitude differences expected from reddening SN $2011 \mathrm{fe}$ using the CCM $R_{V}=1.4$ extinction law with $E(B-V)=1.4$. The solid line shows the magnitude difference expected from circumstellar scattering of dust with $n_{h}=10^{22.4}$ and $r=10^{16} \mathrm{~cm}$. Rather than the monotonic decrease in extinction expected from circumstellar scattering, the SN2014J-SN2011fe differences increase slightly to maximum light. A better match between the observed magnitude differences and that expected from pure interstellar scattering can be achieved by scaling the epochs of SN 2011fe by a factor of 1.1. To mimic the early decrease in $A_{\lambda}$ from scattering one can assume the intrinsic light curve was broader. After maximum, however, $A_{\lambda}$ would increase as SN 2014J fades faster than the assumed light curve.

interpolation and subtraction. This results in a premaximum decrease in $\boldsymbol{A}_{\lambda}$ but also a post-maximum increase in $\boldsymbol{A}_{\lambda}$ as the post-maximum light curve shape should be stretched out much more than SN 2014J.

Interestingly, Foley et al. (2014) do find a temporal change in $R_{V}$ when comparing UV/optical spectra of SN $2014 \mathrm{~J}$ to SNe 
2011fe and 2013dy. They cite Patat (2005) in claiming this as evidence of circumstellar scattering. But the temporal change found by Foley et al. (2014) is a positive increase in $R_{V}$, while Patat (2006; Figure 13) and Wang (2005) predict a temporal decrease in $R_{V}^{0} . R_{V}$ is fairly flat in time, as $A_{V}$ and $E(B-V)$ are both decreasing. The temporal difference in extinction curves, which increases in the blue (Figure 12; Foley et al. 2014), is also cited in support of the circumstellar scattering. However, the predictions of Patat et al. (2006) show such a difference between 6000 and $4000 \AA$ (Figure 14), while Foley et al. (2014) see the difference only growing shortward of $4000 \AA$. The UV region is known to be a source of increased variation in SNe Ia (Brown et al. 2010; Foley \& Kirshner 2013; Milne et al. 2013).

\subsection{Direct Comparison with Light Curves}

The change in apparent extinction discussed above is really just the effect of scattered light being added to the observed flux and slowing down the post-maximum decline. This increased flux is called a light echo at later times (see e.g., Patat 2005) but the effect is the same. The change in light curve shape near maximum light was also studied by Amanullah \& Goobar (2011) for scattering clouds at various radii. The magnitude of the effect is smaller at smaller radii because the light is scattered back into the LOS with a short delay time. The effect is smaller at larger radii because the photons have a much larger delay time and are more spread out in time. Dust corresponding to $E(B-V) \sim 0.4$ at radii between $10^{16}$ and $10^{19} \mathrm{~cm}$ is expected to broaden the light curve such that the measured $\Delta m_{15}(B)$ is increased by up to several tenths of a magnitude (Amanullah \& Goobar 2011). Their simulations broaden a normal SN with $\Delta m_{15}(B)=1.05$ to $\Delta m_{15}(B)=0.4$, broader than ever observed. To result in the rather normally broad observed $\Delta m_{15}(B)=1.1$, SN 2014J would have to have been intrinsically narrow, inconsistent with the observed highvelocity features, otherwise spectroscopic similarity to SN 2011fe and SN 2007co (Foley et al. 2014), and the absolute magnitudes. Comparing to other Swift $\mathrm{SNe}$ or with the SN 2011fe with different stretch values applied, one can obtain magnitude differences which decrease after maximum light but which are accompanied by a rise before maximum light. One would have to assume that SN 2014J had an intrinsically long rise time and an intrinsically fast decay in order for scattering to result in the light curve observed.

We now directly compare our scattering models to the observed light curve. The scattering model described here is dependent on the hydrogen column density (nh; as discussed above, this is used to calculate the optical depth based on the dust model of Weingartner \& Draine 2001) and the radius $(r)$ of the dust shell (in our analytic approximation we consider only a thin shell). An additional uncertainty is how much of the reddening is due to circumstellar rather than interstellar dust. We certainly expect significant interstellar dust in the starburst host M82 (Hutton et al. 2014). Multiple lines from sodium, potassium, and calcium (Foley et al. 2014; Goobar et al. 2014; Graham et al. 2014) as well as strong diffuse interstellar bands were detected in SN 2014J spectra (Welty et al. 2014), also suggestive of interstellar dust.

Foley et al. (2014) suggested half of the extinction is from interstellar dust. We ran many simulations of the SN $2011 \mathrm{fe}$ spectral series being scattered by many configureations of dust distance and column density. This was followed by interstellar reddening using the CCM $R_{V}=2.6$ law with $E(B-V)=0.45$, as suggested by Foley et al. (2014), and the small amount of MW reddening using the CCM $R_{V}=3.1$ law and $E(B-V)=0.054$. In the left panel of Figure 5 we show the observed photometry to different models for the scattering light curve. Of interest are the light curve shapes and the relative offsets between the bands (which represents the colors). While the light curve shapes can be matched with a smaller radius $\left(10^{16} \mathrm{~cm}\right)$, the colors do not match for any model and $R_{V}$ is too high. Furthermore, scattering from nearby dust within $10^{17} \mathrm{~cm}$ appears to be ruled out due to the lack of infrared emission (Johansson et al. 2014). We also plot the $B-V$ color evolution and, by comparison with SN 2011fe, the $E(B-V), R_{V}$, and $R_{V}^{0}$ evolution. Here we are using the $B$ and $V$ data from Marion et al. (2015).

As shown above in Figure 4, changing the intrinsic colors and light curve shapes and then adding circumstellar scattering could reproduce the observed light curve shapes. However, the excellent matches of SN 2011fe reddened by a foreground screen of dust, albeit with extreme $R_{V}$, to the observations of SN 2014J suggest that such fine tuning is not the answer. In the right panel of Figure 5 we show SN 2011fe extinguished by a CCM extinction law with $R_{V}=1.4$ and $E(B-V)=1.4$ and the small amount of MW reddening using the CCM $R_{V}=3.1$ law and $E(B-V)=0.054$. We also show a combination of CCM $R_{V}=2.6$ law with $E(B-V)=0.45$ and a power-law extinction curve similar to Goobar (2008) with $a=0.83, p=-2.6$ and $E(B-V)=0.6$ (and the MW reddening) suggested by Foley et al. (2014). Both give a reasonable match. The $R_{V}$ for the Foley et al. (2014) combination model is higher, but that is a derived parameter from $A_{V}$ and $B-V$, neither of which are significantly worse than the CCM 1.4 model, $B-V$ is simply lower instead of higher. Agreement is not perfect in the wide range of observational data (Amanullah et al. 2014; Foley et al. 2014), but there is an intrinsic dispersion in the colors of $\mathrm{SNe}$ Ia which could affect our inferred reddening values. We may also be trying to force an extinction law into particular functional forms which it may not follow. As discussed above, the fact that some or all of the extinction law can be fit with a power law does not mean it is circumstellar in origin. But the fitting of the extinction curve with a component of dust with a larger $R_{V}$ more similar to the LMC or the MW does reduce the amount of dust which would need to be peculiar. It is likely the case that we do not understand the dust in external galaxies as well as we think we do.

\section{GALAXY EXTINCTION}

If the dust extinction to SN 2014J is interstellar, rather than circumstellar, than why does it seem so different than MW dust? Much of the effort of explaining the extinction laws toward SNe Ia has assumed it was the SN extinction which is peculiar, requiring differences in the dust geometry or the intrinsic colors of SNe Ia. However, one could also examine the methods by which galaxy reddening laws are determined. Maybe a large fraction of external galaxies have "peculiar" dust.

While lines of sight in the MW, LMC, and SMC can be probed by comparing single stars to low reddening counterparts, the extinction laws in other galaxies are usually inferred from the integrated light of stellar populations. As in the reddening study of Hutton et al. (2014) for M82, the dust correction is often done assuming a foreground sheet of dust. 

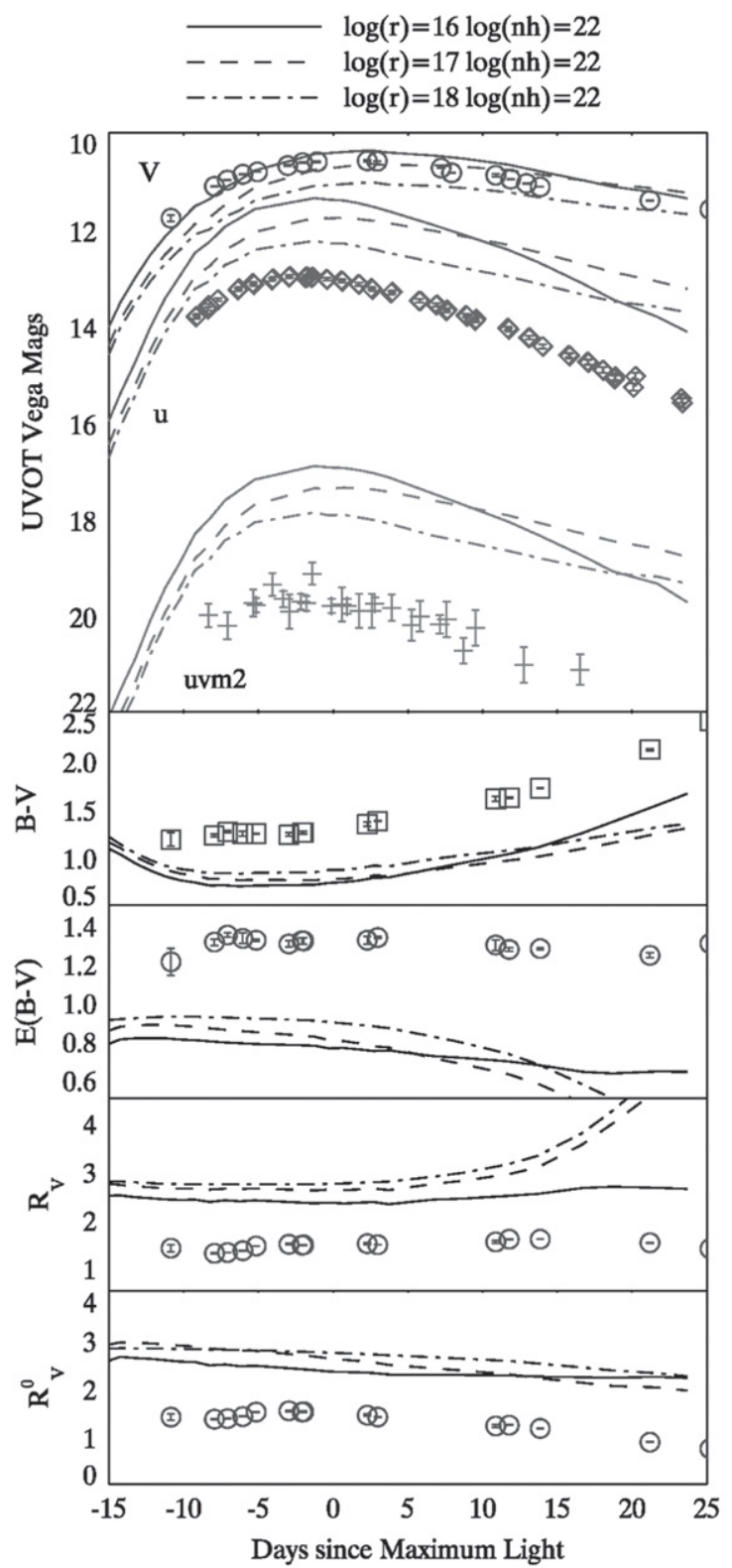

- SN2011fe CCM 1.4

SN2011fe CSMD

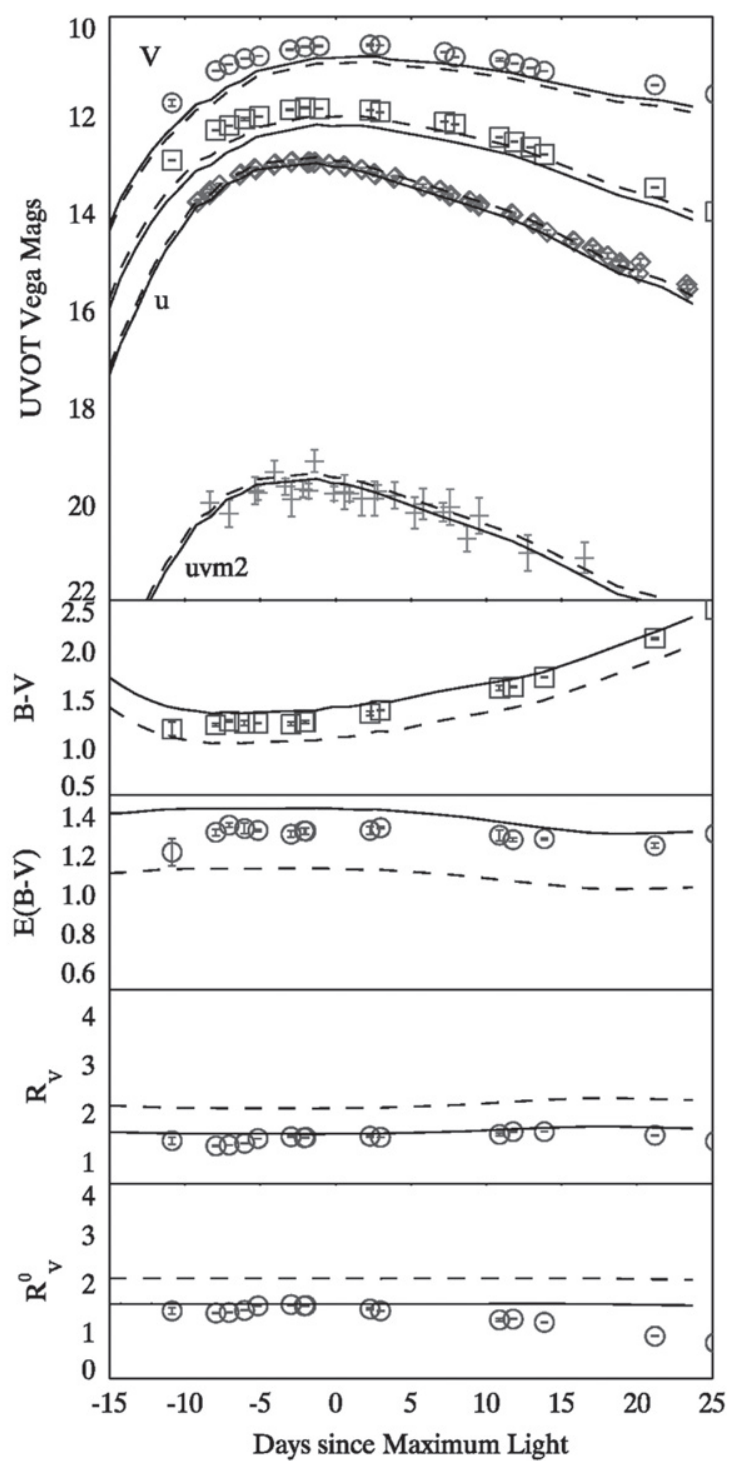

Figure 5. Left: The SN2014J photometry, $B-V$ color, $E(B-V)$ color excess (compared to SN 2011fe), $R_{V}$ (being the time varying $A_{V}$ compared to SN $2011 \mathrm{fe}$ and the time-varying $E(B-V))$ and $R_{V}^{0}$ are compared to models with the SN 2011fe series modified by circumstellar dust at different radius values and a foreground reddening of $E(B-V)=0.45$ and $R_{V}=2.6$ as suggested by Foley et al. (2014). The Milky Way reddening of $E(B-V)=0.054$ and $R_{V}=3.1$ is used in the model. When approximately matching the $\mathrm{V}$ band magnitude, it is hard to match the other filters, colors, or $R_{V}$. Right: the SN2014J photometry, $B-V$ color, $E(B-V), R_{V}$, and $R_{V}^{0}$ are compared to a model with SN 2011fe reddened with a Cardelli law with $E(B-V)=1.4$ and $R_{V}=1.4$. We also show the predictions from the Foley et al. (2014) combination of a Cardelli law with $E(B-V)=0.45$ and a more reasonable $R_{V}=2.6$ with a power-law extinction curve similar to Goobar (2008) with $a=0.83$, $p=-2.6$, and $E(B-V)=0.6$ (called CSMD).

Such an assumption is depicted in the left panel of Figure 6. In reality, the dust is likely mixed in more continuously with the stars. To test the effect such a change in the geometry would have on the inferred dust extinction law, we show two additional scenarios. In the first case, four stars (using Vega as the spectral template) are extinguished by four clouds of dust with a combined $E(B-V)=1$ in the foreground. The extinction is computed using a Cardelli law with $R_{V}=1.7$. A value of $R_{V}=1.8$ would be measured, not too different from the input value. The second scenario mixes the stars and dust evenly, such that one star is extinguished by one cloud, one star by two, one star by three clouds, and one star by four clouds. The effective extinction law is determined by comparing the total transmitted flux to the unextinguished flux of the four stars. The effective extinction has $R_{V}=2.1$. The third scenario has the dust content increasing more rapidly than the stellar content such that one star is extinguished by one cloud, one by two, one by four clouds, and one by eight clouds. Assuming that the four stars are extinguished only be foreground dust, the effective extinction has $R_{V}=2.7$. So mixing the dust in with the stars increases the effective $R_{V}$ above what one would measure if it was truly just a foreground dust screen. This difference in shape is due to differences in the total extinction as well as the difference in extinction between the $\mathrm{B}$ and $\mathrm{V}$ bands which are 

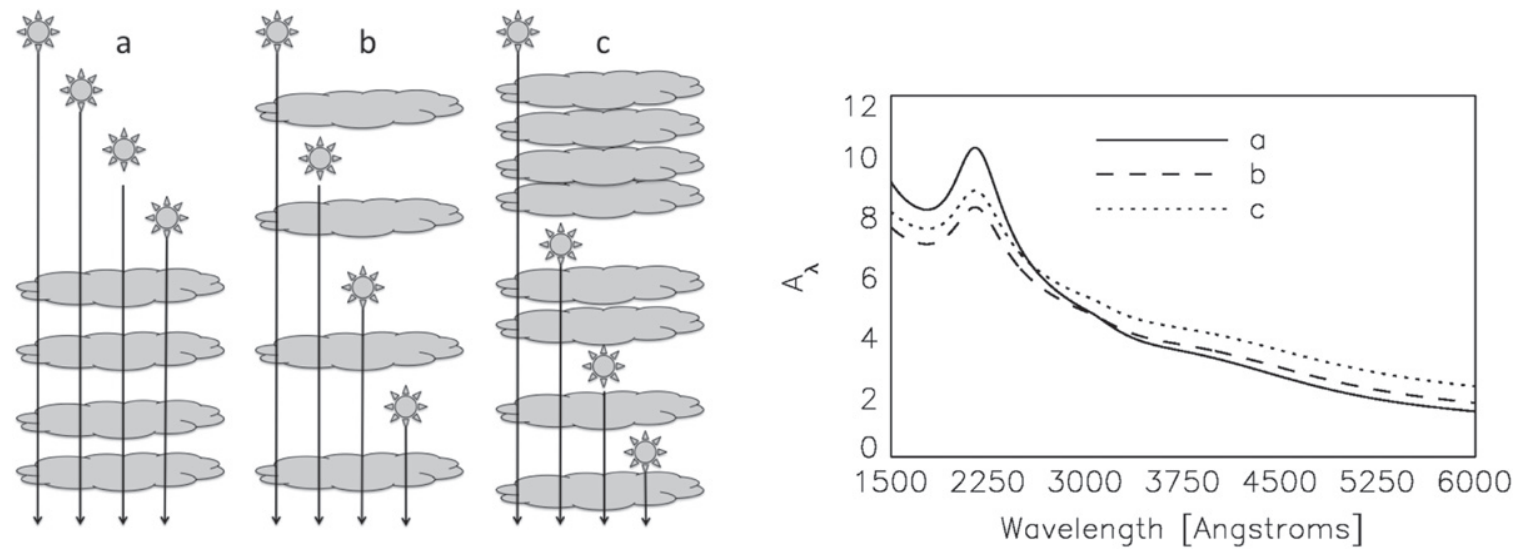

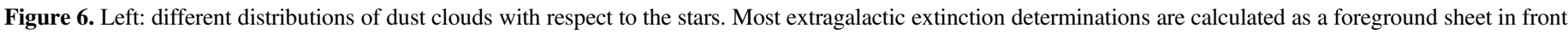

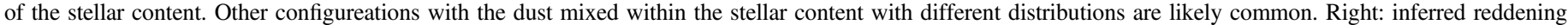

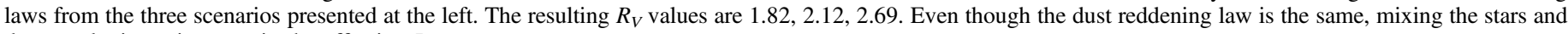
dust results in an increase in the effective $R_{V}$.

used to normalize the curve. The contribution of scattering was ignored, even along our one-dimensional LOS. The real situation is even more complicated.

This test was done with a known spectrum for the intrinsic light. In practice, the stellar mass, effective age or star formation history, and extinction are all being solved for simultaneously. Even knowing the intrinsic stellar content, assuming a wrong geometry results in an incorrect extinction law. When the intrinsic stellar content is unknown, then assuming that wrong geometry is unlikely to result in correct stellar masses, ages, and extinction in much more complicated situations. Stellar population synthesis models should explore the variation caused by different but reasonable assumptions on the dust distribution on the inferred parameters. Physically motivated dust prescriptions, such as that determined by Witt et al. (1992) or Charlot \& Fall (2000), could improve our understanding. They may also reveal that dust in other galaxies is not as similar to MW dust as has been assumed thus far.

Patat et al. (2014) have studied the polarization to several reddened $\mathrm{SNe}$ and found that they do not follow the relations determined for stars in the MW. Studying the extinction and polarization laws in external galaxies using $\mathrm{SNe}$ is similar to the star-matching methods used in the MW. The circumstellar scattering arguments (Wang 2005; Goobar 2008; Foley et al. 2014) have tried to explain the apparently different colors of reddened $\mathrm{SNe}$ with normal dust but an unusual geometry. However, it may be the dust itself which is unusual, at least compared to what we understand. A more fundamental point, and one addressed much better by Calzetti (2001), is that "dust obscuration of galaxies is conceptually different from the dust extinction of stars," and the methods used are very different too. By comparing a single reddened SN with a similar unreddened comparison, we can probe dust extinction in external galaxies in a similar manner to how MW dust extinction laws are probed. This is especially the case for SN 2014J, because the multi-epoch, multi-wavelength comparisons can be done with different subsets and assumptions as shown well by Amanullah et al. (2014) and Foley et al. (2014). Further data on both reddened and unreddened SNe (since we need comparison objects spanning the properties of the reddened $\mathrm{SNe}$ ) will improve our knowledge of more galaxies beyond our own.

\section{SUMMARY}

We have shown that the effective reddening law is dependent on the dust geometry, column density and phase. This corresponds to the change in extinction, and light curve predicted by Wang (2005) and Amanullah \& Goobar (2011). Thus the same reddening law is not applicable to all situations. The reddening law for a specific dust geometry and epoch may be fit with a power law, but simply matching a power law does not mean that the reddening is from circumstellar dust. A full scattering model should specify the geometry and optical depth.

By comparing the observed NUV-optical spectra of SN 2014J with SNe 2011fe and 2012fr, we spectroscopically confirm a low value of $R_{V}$, consistent with that found by others Amanullah et al. (2014), Foley et al. (2014), Goobar et al. (2014), Marion et al. (2015). The light curve shapes can be well modeled by reddening the spectral series of SN 2011fe, showing no evidence for contribution from scattered light. By comparison with models for the temporal signatures of circumstellar scattering, we conclude that little or none of the dust extinction comes from circumstellar dust. Very nearby dust which would steepen the extinction curve without significantly changing the light curve shapes is ruled out by the lack of infrared emission (Johansson et al. 2014). This is also consistent with the clean circumstellar environment suggested by the radio (Pérez-Torres et al. 2014) and X-ray (Margutti et al. 2014) limits and the lack of variation in the narrow sodium absorption lines (Foley et al. 2014). While circumstellar dust could still modify the extinction to some $\mathrm{SNe}$ Ia, it does not appear to be the cause of the low $R_{V}$ for SN 2014J. It has been shown in Wang (2005) and Amanullah \& Goobar (2011) that circumstellar dust between $10^{16}$ and $10^{20} \mathrm{~cm}$ has a significant broadening effect on the observed light curve. Here we confirm the Wang (2005) finding that the low $R_{V}$ near maximum light occurs only at radii less than $10^{17} \mathrm{~cm}$ where the delay time from scattering is low. If circumstellar scattering is pursued as an explanation for the low $R_{V}$ of individual or groups of $\mathrm{SNe}$ without broadened light curves, than the inner radius at which dust is not destroyed (currently estimated to be $10^{16} \mathrm{~cm}$; Amanullah \& Goobar 2011, see also Pearce \& Mayes 1986) should be more carefully calculated. Since a geometric cause of the low $R_{V}$ appears to be 


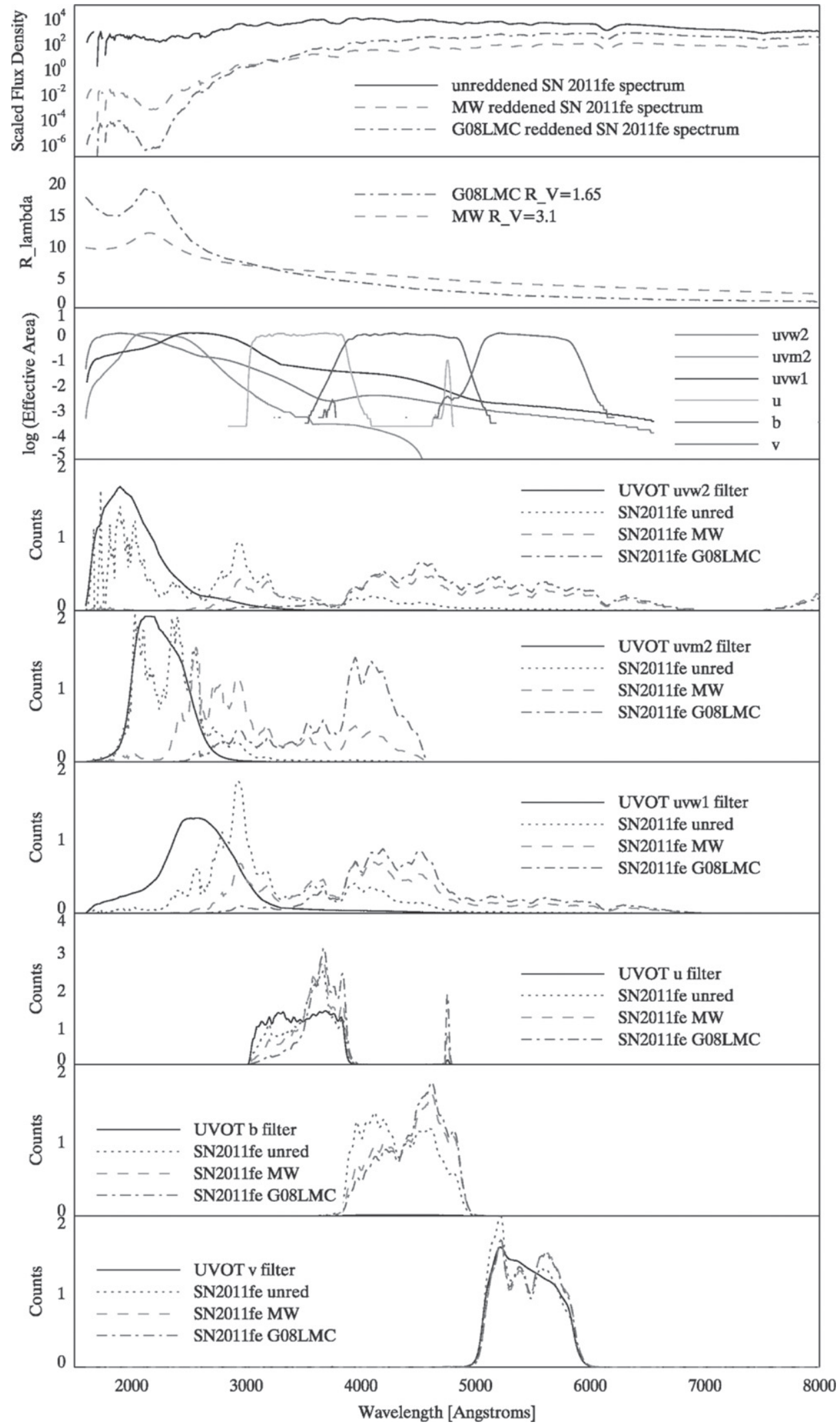

Figure 7. Top panel: HST UV spectrum of SN2011fe near maximum light: unreddened, and with a Milky Way (MW: $\left.R_{V}=3.1\right)$ and an LMC extinction law with circumstellar scattering (G08LMC)(Goobar 2008; Brown et al. 2010) for an $E(B-V)=1.2$. Second panel: wavelength dependence of the MW and G08LMC extinction coefficient. Third panel: UVOT filter effective area curves. Bottom panels: count spectra after passing the unreddened and reddened spectra through the UVOT filter curves and normalized by the total count rate. 


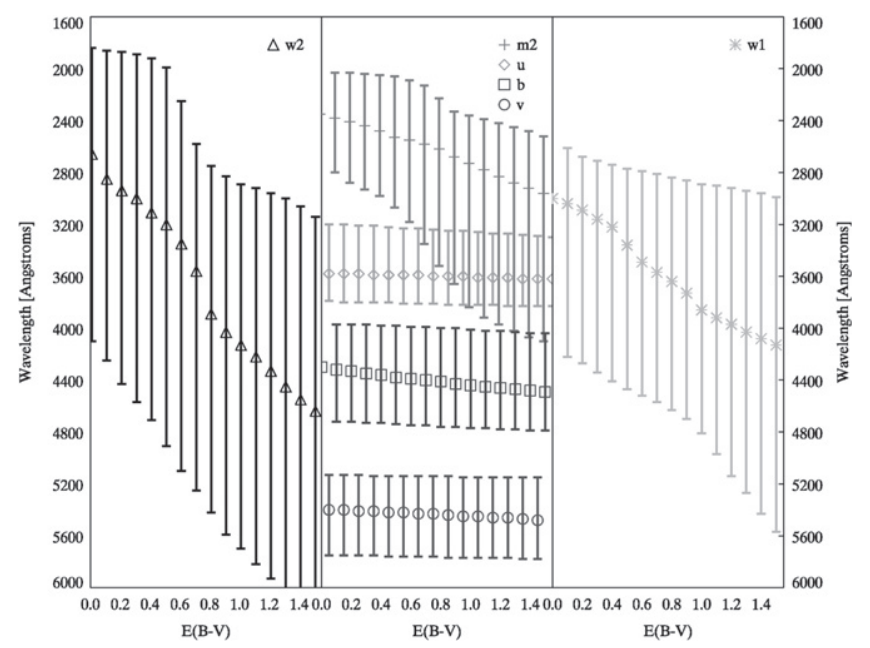

Figure 8. Wavelength ranges of the photons transmitted through the UVOT filters for the SN $2011 \mathrm{fe}$ spectrum as a function of reddening (using an MW extinction law with $R_{V}=3.1$ ). The symbols represent the effective wavelength of the filter for the reddened spectrum, defined as the wavelength at which half of the detected photons are received from shorter and longer wavelengths. The vertical error bars representing the wavelengths between $10 \%$ and $90 \%$ of the transmitted photons (i.e., the region within those error bars contain $80 \%$ of the transmitted photons).

ruled out for SN $2014 \mathrm{~J}$, the size and chemistry of grains which could cause it should also be further studied.

The high reddening of SN 2014J allowed detailed studies of the NUV, optical, and NIR extinction which were less affected by the intrinsic differences between SNe. However, it also complicated mid-UV photometry that could otherwise probe the region around the $2175 \AA$ bump in many extinction curves. Probing shorter wavelengths will require mid-UV photometry of less reddened SNe where the effective wavelength is not so dramatically shifted and spectroscopy as distance and sensitivity allow. The intrinsic differences will also have to be more carefully accounted for, as the variation in SNe Ia increases dramatically in the mid-UV (Brown et al. 2010; Foley \& Kirshner 2013; Milne et al. 2013). More spectral sequences of $\mathrm{SNe}$ Ia in the UV are needed to disentangle the effects of intrinsic and extinction differences in understanding SN colors.

P.J.B. is supported by the Mitchell Postdoctoral Fellowship and NASA ADAP grant NNX13AF35G. L.W. acknowledges support from NSF grant AST-0708873. P.A.M. acknowledges support from NASA ADAP grant NNX10AD58G. A.B., M.P., and N.P.M.K. acknowledge the support of the UK Space Agency. This work made use of public data in the Swift data archive and the NASA/IPAC Extragalactic Database (NED), which is operated by the Jet Propulsion Laboratory, California Institute of Technology, under contract with NASA.

\section{APPENDIX FILTER COMPLICATIONS}

To properly interpret photometric data, including light curves, colors, and the extinction through a given filter, it is important to understand what is being measured in a photometric data point. In a count-based detector, all incoming photons resulting in a detected count are treated the same, regardless of wavelength/energy. So the spectral shape of the source (including reddening) and the total filter + telescope + atmosphere transmission (as appropriate for the instrument) are equally important. The effect of the optical tails of the uvw2 and uvw1 filters is already recognized (Poole et al. 2008), especially for the very red spectrum of a SNe Ia (Brown et al. 2010). The severe reddening to SN 2014J exacerbates the problems and makes interpretation particularly complicated. To illustrate the effects, in Figure 7 we use the HST STIS spectrum of SN 2011fe from 2011 September 13 (the epoch with the broadest wavelength coverage; Mazzali et al. 2014) and apply a reddening of $E(B-V)$ using the MW 3.1 and G08LMC extinction laws, both with an $E(B-V)=1.2$. Also shown are the UVOT filter curves and the resulting count spectra when the unreddened and reddened SN spectra are passed through the UVOT filter curves. The count spectra are normalized by the total number of counts for that spectrum/filter combination (and then scaled uniformly for presentation purposes) to highlight the distribution of the photons with wavelength.

The reddening of the spectrum serves to shift the distribution of observed photons to longer wavelengths. The effect is small in the optical bands but increases as the wavelength range of the filter decreases. This general effect is due to the steepening of the extinction laws at shorter wavelengths. For the UVOT UV filters, the non-negligible tails of the filters dramatically increase the magnitude of the effect. At a wavelength of $3500 \AA$ the uvw2 and uvw1 filters still have about $10 \%$ and $1 \%$ of their peak transmission. This is already important for an unreddened SN Ia, as they typically have one hundred times more flux at $4000 \AA$ than they do at $2000 \AA$. While the intrinsically very red SN Ia spectrum already has significant counts transmitted through the optical tails of the uvw2 and uvw1 filters, the G08LMC law in particular completely suppresses the remaining mid-UV and NUV flux, resulting in only optical (B band) counts being transmitted. The uvm2 filter has a better cut off, with only $0.1 \%$ of the peak transmission at $3500 \AA$, but, as shown in Figure 7, this could still be significant if the observed source is red enough.

To further quantify this, Figure 8 plots the effective wavelength (essentially the median point for the detected photons for a given filter/spectrum combination) for the Swift UVOT filters for the SN 2011 fe spectrum with various amounts of reddening applied. The wavelengths bounding the central $80 \%$ of the photons are represented by the vertical error bars. The wavelength ranges of the observed photons for the optical filters are rather constant with reddening due to their sharp cut offs in transmission. The UV filters behave very differently. The optical tails of the uvw2 and uvw1 filters cause the detected photons to have a large range in wavelength which continues to shift to longer wavelengths with increased reddening. The effective wavelengths for uvw2 and uvw1 match the $\mathrm{u}$ band for a reddening of $E(B-V)=0.7$ and continue to grow. Because of the above issues, our analysis above excluded the uvw2 and uvw1 filters and focused on the other UVOT filters: uvm2, u, b, and v.

The uvm2 filter does not have the optical tails of the other UV filters nor does it cut off quite as sharply as the optical filters. The effective wavelength begins at $2350 \AA$ for an unreddened SN Ia and increases slowly to approach $3000 \AA$ for $E(B-V)=1.2$. So while its effective transmission shifts to longer wavelengths into a broader NUV filter, more than half the photons are still expected to be from wavelengths shorter than the atmospheric cut off (for a MW 3.1 extinction law). 
Clearly the extinction in a given UV band cannot be accurately represented by a magnitude difference at the effective wavelength, as is often done in the optical and NIR (e.g., Figure 14 in Folatelli et al. 2010 and Figure 11 in Wang et al. 2008). To properly interpret the data, one must compare to spectral templates which have been appropriately reddened. Spectrophotometry of such models will include the effects of the filters shapes (assuming the filter curves are accurately determined) and allow a fair comparison.

\section{REFERENCES}

Amanullah, R., \& Goobar, A. 2011, ApJ, 735, 20

Amanullah, R., Goobar, A., Johansson, J., et al. 2014, ApJL, 788, L21

Breeveld, A. A., Landsman, W., Holland, S. T., et al. 2011, in AIP Conf. Ser.

1358, Gamma Ray Bursts 2010, ed. J. E. McEnery, J. L. Racusin, \& N. Gehrels (Melville, NY: AIP), 373

Brown, P. J., Breeveld, A. A., Holland, S., Kuin, P., \& Pritchard, T. 2014a, Ap\&SS, 354, 89

Brown, P. J., \& Evans, P. A. 2014, ATel, 5809, 1

Brown, P. J., Dawson, K. S., de Pasquale, M., et al. 2012, ApJ, 753, 22

Brown, P. J., Holland, S. T., Immler, S., et al. 2009, AJ, 137, 4517

Brown, P. J., Kuin, P., Scalzo, R., et al. 2014b, ApJ, 787, 29

Brown, P. J., Roming, P. W. A., Milne, P., et al. 2010, ApJ, 721, 1608 Calzetti, D. 2001, PASP, 113, 1449

Cao, Y., Kasliwal, M. M., McKay, A., \& Bradley, A. 2014, ATel, 5786, 1

Cardelli, J. A., Clayton, G. C., \& Mathis, J. S. 1989, ApJ, 345, 245

Charlot, S., \& Fall, S. M. 2000, ApJ, 539, 718

Childress, M. J., Scalzo, R. A., Sim, S. A., et al. 2013, ApJ, 770, 29

Conley, A., Carlberg, R. G., Guy, J., et al. 2007, ApJL, 664, L13

Elias-Rosa, N., Benetti, S., Cappellaro, E., et al. 2006, MNRAS, 369, 1880

Folatelli, G., Phillips, M. M., Burns, C. R., et al. 2010, AJ, 139, 120

Foley, R. J., \& Kirshner, R. P. 2013, ApJL, 769, L1

Foley, R. J., Sanders, N. E., \& Kirshner, R. P. 2011, ApJ, 742, 89

Foley, R. J., Fox, O. D., McCully, C., et al. 2014, MNRAS, 443, 2887

Fossey, J., Cooke, B., Pollack, G., Wilde, M., \& Wright, T. 2014, CBET, 3792,1

Freedman, W. L., Madore, B. F., Gibson, B. K., et al. 2001, ApJ, 553, 47

Gehrels, N., Chincarini, G., Giommi, P., et al. 2004, ApJ, 611, 1005

Goldhaber, G., Groom, D. E., Kim, A., et al. 2001, ApJ, 558, 359

Goobar, A. 2008, ApJL, 686, L103

Goobar, A., Johansson, J., Amanullah, R., et al. 2014, ApJL, 784, L12

Gordon, K. D., Clayton, G. C., Misselt, K. A., Landolt, A. U., \& Wolff, M. J. 2003, ApJ, 594, 279

Graham, M. L., Valenti, S., Fulton, B. J., et al. 2015, ApJ, 801, 136G
Henyey, L. G., \& Greenstein, J. L. 1941, ApJ, 93, 70

Hicken, M., Wood-Vasey, W. M., Blondin, S., et al. 2009, ApJ, 700, 1097

Hsiao, E. Y., Marion, G. H., Phillips, M. M., et al. 2013, ApJ, 766, 72

Hutton, S., Ferreras, I., Wu, K., et al. 2014, MNRAS, 440, 150H

Jacobs, B. A., Rizzi, L., Tully, R. B., et al. 2009, AJ, 138, 332

Johansson, J., Goobar, A., Kasliwal, M. M., et al. 2014, arXiv:1411.3332J

Kessler, R., Becker, A. C., Cinabro, D., et al. 2009, ApJS, 185, 32

Krisciunas, K., Prieto, J. L., Garnavich, P. M., et al. 2006, AJ, 131, 1639

Kuin, N. P. M. 2014, ascl soft, (record ascl:1410.004).

Kuin, N. P. M., Landsman, W., Breeveld, A. A., et al. 2015, MNRAS, 449, $2514 \mathrm{~K}$

Margutti, R., Parrent, J., Kamble, A., et al. 2014, ApJ, 790, 52

Marion, G. H., Sand, D. J., Hsiao, E. Y., et al. 2015, ApJ, 798, 39

Mathis, J. S. 1972, ApJ, 176, 651

Mazzali, P. A., Sullivan, M., Hachinger, S., et al. 2014, MNRAS, 439, 1959

McCall, M. L. 2004, AJ, 128, 2144

Milne, P. A., Brown, P. J., Roming, P. W. A., Bufano, F., \& Gehrels, N. 2013, ApJ, 779, 23

Milne, P. A., Brown, P. J., Roming, P. W. A., et al. 2010, ApJ, 721, 1627

Munari, U., Henden, A., Belligoli, R., et al. 2013, NewA, 20, 30

Patat, F. 2005, MNRAS, 357, 1161

Patat, F., Benetti, S., Cappellaro, E., \& Turatto, M. 2006, MNRAS, 369, 1949

Patat, F., Taubenberger, S., Cox, N. L. J., et al. 2014, arXiv:1407.0136P

Pearce, G., \& Mayes, A. J. 1986, A\&A, 155, 291

Pereira, R., Thomas, R. C., Aldering, G., et al. 2013, A\&A, 554, A27

Pérez-Torres, M. A., Lundqvist, P., Beswick, R. J., et al. 2014, ApJ, 792, 38 Phillips, M. M. 1993, ApJL, 413, L105

Phillips, M. M., Lira, P., Suntzeff, N. B., et al. 1999, AJ, 118, 1766

Poole, T. S., Breeveld, A. A., Page, M. J., et al. 2008, MNRAS, 383, 627

Reindl, B., Tammann, G. A., Sandage, A., \& Saha, A. 2005, ApJ, 624, 532

Reiss, A. G., Press, W. H., \& Kirshner, R. P. 1996, ApJ, 473, 88

Roming, P. W. A., Kennedy, T. E., Mason, K. O., et al. 2005, SSRv, 120, 95

Shappee, B. J., \& Stanek, K. Z. 2011, ApJ, 733, 124

Tripp, R. 1998, A\&A, 331, 815

Tsvetkov, D. Y., Metlov, V. G., Shugarov, S. Y., Tarasova, T. N., \& Pavlyuk, N. N. 2014, CoSka, 44, 67

Wang, L. 2005, ApJL, 635, L33

Wang, X., Wang, L., Pain, R., Zhou, X., \& Li, Z. 2006, ApJ, 645, 488

Wang, X., Li, W., Filippenko, A. V., et al. 2008, ApJ, 675, 626

Wang, X., Filippenko, A. V., Ganeshalingam, M., et al. 2009, ApJL, 699, L139

Weingartner, J. C., \& Draine, B. T. 2001, ApJ, 548, 296

Welty, D. E., Ritchey, A. M., Dahlstrom, J. A., \& York, D. G. 2014, ApJ, 792, 106

Witt, A. N., Thronson, H. A., Jr., \& Capuano, J. M., Jr. 1992, ApJ, 393, 611 Yaron, O., \& Gal-Yam, A. 2012, PASP, 124, 668

Zhang, J.-J., Wang, X.-F., Bai, J.-M., et al. 2014, AJ, 148, 1 